\title{
GAIA Level 2 Neonatal Death in a Term Live Birth
}

National Cancer Institute

\section{Source}

National Cancer Institute. GAIA Level 2 Neonatal Death in a Term Live Birth. NCI

Thesaurus. Code C127979.

GAIA Level 2 Neonatal Death in a Term Live Birth is defined by three criteria: first, the infant must be live born; second, at least one of the following requirements must be met:

a) Gestational age greater than or equal to 37 weeks (where the GA level of certainty is either level one or level two) OR b) Birth weight greater than or equal to 2500g; third, the death of the infant within the first 28 days of life must be documented. 